\title{
Beyond small-angle x-ray scattering: Exploiting angular correlations
}

\author{
D. K. Saldin, ${ }^{1}$ H. C. Poon, ${ }^{1}$ V. L. Shneerson, ${ }^{1, *}$ M. Howells, ${ }^{2}$ H. N. Chapman, ${ }^{3}$ R. A. Kirian, ${ }^{4}$ \\ K. E. Schmidt, ${ }^{4}$ and J. C. H. Spence ${ }^{4}$ \\ ${ }^{1}$ Department of Physics, University of Wisconsin-Milwaukee, Milwaukee, Wisconsin 53211, USA \\ ${ }^{2}$ Lawrence Berkeley National Laboratory, Berkeley, California 94720, USA \\ ${ }^{3}$ Center for Free Electron Laser Science, DESY, Universität Hamburg, 22607 Hamburg, Germany \\ ${ }^{4}$ Department of Physics, Arizona State University, Tempe, Arizona 85287, USA
}

(Received 18 March 2010; published 7 May 2010)

\begin{abstract}
We propose a method for determining the projected electron density of an individual molecule from diffraction patterns of many such simultaneously illuminated molecules, randomly oriented about an axis parallel to an incident x-ray beam. We illustrate the idea with a simulation of a structure determination of a K-channel membrane protein in situ.
\end{abstract}

DOI: 10.1103/PhysRevB.81.174105

PACS number(s): 87.64.Bx, 61.05.cp, 82.53.Ps

\section{INTRODUCTION}

The atomic-scale structure of a molecule is generally determined by the analysis of the distribution of scattered $x$ rays or electrons. However, the sustained x-ray or electron fluence needed for image reconstruction would destroy all high-resolution detail if focused on a single molecule since resolution varies inversely as the third or fourth power of fluence. ${ }^{1}$ If the molecules form crystals, the scattered intensities may be greatly amplified by sharing this fluence over many identical molecules, while allowing the reconstruction of the periodically averaged undamaged charge density of a molecule. Unfortunately, not all molecules of interest do form crystals, and even if crystallization were possible, the scattered signal would be sampled only at the Shannon "frequency" of the complex amplitudes, resulting in loss of phase information. If the scattered intensities from a single molecule could be measured at their (finer) Shannon angular sampling rate, the structure of the scatterer could be determined by iterative phasing algorithms. ${ }^{2}$ These advantages of signal amplification, damage reduction for high resolution, and access to oversampled intensities (allowing solution of the phase problem) may be combined if the structure of a single particle may be determined from diffraction patterns (DPs) from many identical particles with neither translational nor orientational order.

In this paper, we suggest how this may advance practical applications, such as structure determination of hard to crystallize, but practically important, membrane proteins, which are the targets of some $70 \%$ of today's medicinal drugs. A similar idea has been proposed by $\mathrm{Kam}^{3,4}$ for molecules in solution where their orientations are completely random in three dimensions (3D). We propose here an application to the determination of the structure of a membrane protein in even more of a natural environment than that of molecules dissolved in a liquid.

Consider x-ray scattering from identical molecules in solution. If the scattering is from radiation of pulse length longer than the rotational diffusion time, $\tau$, of the molecules, the signal from each molecule will be its rotational average and signal amplification is provided by the addition of such rotational averages from all illuminated molecules, as ex- ploited by the technique of small-angle $\mathrm{x}$-ray scattering (SAXS), from which information about the molecular shape may be found..$^{5-7}$ Variations in a SAXS signal are greatest in the region of very small magnitude scattering wave vector, $q$, where the signal is sensitive largely to the overall shape of the molecule, rather than its internal structure. ${ }^{8}$

The increasing availability of intense short-pulse x-ray sources allows a measurement of a type not possible before, namely, angular fluctuations of intensity about mean values on rings of constant $q$ from snapshots of a relatively small number of identical particles, differing only in random positions and random orientations. The diffraction patterns may be recorded either from stationary particles or more generally with a recording time shorter than $\tau$. The angular correlation functions of these intensity fluctuations contain information about the multidimensional diffraction volume of the individual scatterers, 3,9 and hence of their internal structure. More intense x-ray sources also allow a useful signal to be measured from higher values of $q$, thus permitting access to higher-resolution structure. Structure reconstruction from these data requires solutions to two phase problems: the first to recover a single-particle diffraction pattern from multiparticle correlations and the second to recover the projected electron density from the reconstructed single-particle diffraction pattern.

\section{THEORY}

We examine here a case where the molecular orientations are assumed random only about a single axis, which is assumed parallel to the incident X-ray beam. As described Section IV, this may model, e.g., a set of a membrane bound protein molecules randomly oriented about an axis normal to a cell membrane in a plane perpendicular to the incident beam.

Assuming a flat Ewald sphere, we represent the scattering vector $\mathbf{q}$ by a vector normal to an $\mathbf{x}$-ray beam, assumed incident normal to the membrane (and therefore parallel to the plane of the membrane) by its polar components $\left(q, \phi_{j}\right)$, where the different azimuthal angles are specified by the subscript $j$, the scattered amplitudes may be represented by the circular harmonic expansion 


$$
A\left(q, \phi_{j}\right)=\sum_{m} A_{m}(q) \exp \left(i m \phi_{j}\right) .
$$

Assuming mutually coherent scattering, the total scattered amplitude from a set of such molecules $k$ with random azimuthal orientations $\omega_{k}$ and random positions $\mathbf{r}_{k}$ is

$$
A\left(q, \phi_{j}\right)=\sum_{k} \sum_{m} A_{m}(q) \exp \left[i m\left(\phi_{j}-\omega_{k}\right)\right] \exp \left(i \mathbf{q} \cdot \mathbf{r}_{k}\right) .
$$

If the resulting scattered intensity at $\left(q, \phi_{j}\right)$ is represented by $I\left(q, \phi_{j}\right)$ and its azimuthal average by $I_{\mathrm{SAXS}}(q)$, the SAXS intensity, then

$$
I\left(q, \phi_{j}\right)-I_{\mathrm{SAXS}}(q)=\sum_{k k^{\prime}} \sum_{m \neq m^{\prime}} A_{m}^{*}(q) \exp \left(-i m \phi_{j}\right) \exp \left(i m \omega_{k}\right) \exp \left(-i \mathbf{q} \cdot \mathbf{r}_{k}\right) A_{m^{\prime}}(q) \exp \left(i m^{\prime} \phi_{j}\right) \exp \left(-i m^{\prime} \omega_{k^{\prime}}\right) \exp \left(i \mathbf{q} \cdot \mathbf{r}_{k^{\prime}}\right)
$$

The subtraction of $I_{\text {SAXS }}(q)$ on the left-hand side (LHS) necessitates the exclusion of the cross terms with $m=m^{\prime}$ on the right-hand side.

The expression (3) enables the evaluation of the angular pair correlations defined by

$$
C_{2}\left(q, q^{\prime} ; \Delta \phi\right)=\left\langle\frac{1}{N_{\phi}} \sum_{j} I^{\prime}\left(q, \phi_{j}\right) I^{\prime}\left(q^{\prime}, \phi_{j}+\Delta \phi\right)\right\rangle_{\mathrm{DP}},
$$

where $N_{\phi}$ is the number of azimuthal angles $\phi_{j}$ at which the intensities are measured and the angular brackets denote an average over DPs,

$$
I^{\prime}\left(q, \phi_{j}\right)=I\left(q, \phi_{j}\right)-I_{\mathrm{SAXS}}(q)
$$

and

$$
I^{\prime}\left(q^{\prime}, \phi_{j}+\Delta \phi\right)=I\left(q^{\prime}, \phi_{j}+\Delta \phi\right)-I_{\mathrm{SAXS}}\left(q^{\prime}\right) .
$$

Substituting Eq. (3) into Eqs. (5) and (6), and the resulting expressions into Eq. (4) and performing the average over the DP yields

$$
C_{2}\left(q, q^{\prime} ; \Delta \phi\right)=N_{p} \sum_{M \neq 0} I_{M}^{*}(q) I_{M}\left(q^{\prime}\right) \exp (i M \Delta \phi),
$$

where $N_{p}$ is the number of particles illuminated per diffraction pattern, and $I_{M}(q)$ the circular harmonic expansion coefficients of the diffracted intensity of a single particle and related to the corresponding expansion coefficients of the scattered amplitude by

$$
I_{M}(q)=\sum_{m} A_{m}^{*}(q) A_{M-m}(q)
$$

It should noted that, although each diffraction pattern is assumed be illuminated by coherent radiation, the interference terms due to the scattering by the different particles are averaged out by the averaging over the DPs in Eq. (4) to leave $C_{2}$ a function of only single-particle quantities. The extraction of the quantities $I_{M}(q)$ would enable the reconstruction of the single-particle DPs.

As a first step toward that end it will be noted that the angular Fourier transforms of the $q$-diagonal pair correlations are

$$
\begin{aligned}
B_{M}(q, q) & =\frac{1}{N_{\phi}} \sum_{\Delta \phi} C_{2}(q, q, \Delta \phi) e^{-i M \Delta \phi}=N_{p} I_{M}(q) I_{M}^{*}(q) \\
& =N_{p}\left|I_{M}(q)\right|^{2} .
\end{aligned}
$$

Thus, just taking the square roots of the quantities $B_{M}(q, q)$ enables the determination of the magnitudes of the circularharmonic expansion coefficients (up to an unimportant scaling factor) via

$$
\left|I_{M}(q)\right| \propto \sqrt{B_{M}(q, q)} .
$$

It will be noted that a reconstruction of the intensity distribution of a single particle can be performed via the equation

$$
I(q, \phi)=\sum_{M} I_{M}(q) \exp (i M \phi)
$$

where the $I_{M}(q)$ are a set of complex coefficients. Thus, the problem of reconstructing the single-particle diffraction pattern has some analogies to that of recovering the electron density of the scattering object from the intensities of the diffraction pattern. In both cases it is necessary to find the phases associated with the magnitudes of a set of complex numbers. The latter problem is the celebrated phase problem of crystallography, whose solution has led to at least one Nobel Prize. ${ }^{10}$ More recently, this area of work has been rejuvenated by the development of iterative phasing algorithms ${ }^{2}$ which work by alternately satisfying constraints in real and reciprocal space.

\section{PHASING THE CIRCULAR-HARMONIC EXPANSION COEFFICIENTS}

$\mathrm{Kam}^{4}$ suggested that the phases of the $I_{M}(q)$ coefficients may be found by constructing from the experimental data triple angular correlations since their angular Fourier transforms are sensitive to the phases. In a previous paper, ${ }^{11}$ we have demonstrated the effectiveness of such an approach for a diffraction pattern with a mirror line, when the $I_{M}(q)$ coefficients become real, so their only ambiguity is one of sign. In this case, the correct combination of signs may be found by an exhaustive search through all possible sign combinations. For diffraction patterns without such a mirror line, 
however, it is necessary to find a set of real numbers between 0 and $2 \pi$ representing the unknown phases. In principle, this may be solved by a global optimization routine such as simulated annealing but we found such a process to be very time consuming and not so reliable. We suggest here a distinct approach to finding these phases in an analogy with the iterative phasing algorithms referred to above.

First note that, in general, the angular Fourier transform of Eq. (7) may be written as

$$
B_{M}\left(q, q^{\prime}\right)=\frac{1}{N_{\phi} \Delta \phi} \sum_{2}\left(q, q^{\prime}\right) \exp (-i M \Delta \phi)=N_{p} I_{M}(q) I_{M}^{*}\left(q^{\prime}\right) .
$$

Since the LHS is a quantity that may be found from experiment, and the magnitudes $\left|I_{M}(q)\right|$ of all the circular-harmonic expansion coefficients may be determined, the phases of these coefficients corresponding to the same values of $M$ but different values of $q$ are not independent. In fact, there are only $M_{\max }-1$ independent phases that need to be determined (where $M_{\max }$ is the maximum value of the azimuthal quantum number used in the calculations).

The phase recovery process begins by choosing a reference resolution ring, specified by, say $q^{\prime}$. We defined the reference ring to be a central one, half way between the outermost and innermost. The phases of the $I_{M}\left(q^{\prime}\right)$ 's are found by first associating random phases with these coefficients. Equation (11) may then be used to find a first estimate of the intensities of the resolution ring $q^{\prime}$ of the diffraction pattern. The resulting intensities $I\left(q^{\prime}, \phi\right)$ are then sorted by magnitude and the signs (they are real numbers by construction) of a certain fraction, say $5 \%$, of lowest intensity are flipped, according to the prescription of Oszlányi and Süto. ${ }^{12}$ An inverse Fourier transform then yields new (complex) values of the $I_{M}\left(q^{\prime}\right)$ coefficients. The phases of these calculated coefficients are then associated with the amplitudes $\left|I_{M}\left(q^{\prime}\right)\right|$ found from the Fourier transforms of the measured angular correlations via Eqs. (9) and (10). Another (forward) Fourier transform is then performed to find an improved estimate of $I\left(q^{\prime}, \phi\right)$ and the entire process iterated to convergence.

Once the phases of the $I_{M}\left(q^{\prime}\right)$ coefficients have been found by this method, in principle, the phases of the $I_{M}(q)$ coefficients of all other resolution rings $q$ follow from Eq. (12). In practice this may be somewhat unreliable since the phases found by the above procedure for the reference resolution ring may not be perfect. They may be least reliable for values of $M$ for which $\left|I_{M}\left(q^{\prime}\right)\right|$ is small. Therefore, we use this method to fix the phases of $I_{M}(q)$ 's for the nearestneighbor resolution rings $q$, where $\left|I_{M}\left(q^{\prime}\right)\right|$ is not too small. Nevertheless, this is a very important step: without it the correct registries of the intensity distributions $I(q, \phi)$ on different resolution rings could not be found.

Having thus estimated both the amplitudes and phases of the $I_{M}(q)$ coefficients for these values of $M$ (let us denote them by $M^{\prime}$ ) for the neighboring resolution rings $q$, the phases of the remaining values of $M \neq M^{\prime}$ can be found by repeating the above the iterative phasing algorithm to those resolutions rings $q$ in turn, fixing both the amplitudes and phases for $M=M^{\prime}$, and only the amplitudes for $M \neq M^{\prime}$. Us- ing this method of phasing neighboring resolution rings, the phasing process is propagated inwards and outwards to the neighboring resolution rings in turn, using its phased nearestneighbor as reference. Thus the reference phases in each ring are determined by the phases found for its neighboring ring by a combination of Eq. (12) and the flipping algorithm.

The real and imaginary parts $x_{r}(t)$ and $x_{i}(t)$, respectively, of the Fourier transforms of a causal function of the form $X(\omega)$, where $X(\omega)$ is zero for $\omega<0$ are known to be related by Hilbert transforms (e.g., Ref. 13). At least for a diffraction pattern with C2 symmetry, as here, this relationship may be exploited to refine the phases obtained with the flipping algorithm. (Friedel's Law guarantees C2 symmetry for a flat Ewald sphere. In the present case the symmetry of the molecular projection guarantees this even for a curved Ewald sphere.) The (real) intensities $I(q, \phi)$ of each resolution ring $q$ play the role of the function $X(\omega)$ while the real and imaginary parts of its complex Fourier coefficients $I_{M}(q)$ may be identified with $x_{r}(t)$ and $x_{i}(t)$, respectively. Although $I(q, \phi)$ is not strictly a causal function, if the diffraction pattern has $\mathrm{C} 2$ symmetry, it is a twofold redundant function, which implies $I(q, \phi+\pi)=I(q, \phi)$. Thus the nonzero Fourier coefficients $I_{M}(q)$ may be found by taking $I(q, \phi)=0$, e.g., negative $\phi$ (assuming $\phi$ is defined from $-\pi$ to $\pi$ ). This means that the $I_{M}(q)$ 's may be related to Fourier transforms of a causal function and thus their real and imaginary parts are related by Hilbert transforms. In the phasing of the coefficients $I_{M}(q)$ we are faced with a situation where both the amplitudes and phases (and therefore the real and imaginary parts) of the $I_{M}(q)$ 's are known for $M=M^{\prime}$ while only the amplitudes are known for $M \neq M^{\prime}$. By constraining the known portions of the real and imaginary parts of $I_{M}(q)$ an iterative algorithm that repeatedly relates the real and imaginary parts of $I_{M}(q)$ allows the recovery of the real and imaginary parts of all the $I_{M}(q)$ coefficients on convergence. We used this method to refine the initial estimates of the phases of the $I_{M}(q)$ coefficients found by the flipping algorithm above. More details of this procedure will be described elsewhere. ${ }^{14}$

\section{APPLICATION TO MODEL OF K-CHANNEL MEMBRANE PROTEINS IN SITU}

The K-channel protein forms a channel for the microtransport of $\mathrm{K}$ ions through a cell membrane, e.g., in the process of neurotransmission. ${ }^{15}$ To a good approximation, the ion channel has to remain perpendicular to the membrane for it to perform its function. However, the different $\mathrm{K}$-channel molecules in a given membrane may have random angles of orientation about the membrane normal. The analysis above therefore appears well suited to extracting the projected electron density of the individual molecules from diffraction patterns formed by illuminating groups of such randomly oriented membrane-bound molecules in situ.

Figure 1 shows the central part of the diffraction pattern of a single K-channel protein molecule in an orientation with incident $\mathrm{X}$-ray beam parallel to its central pore, as simulated with the structure data in entry $3 \mathrm{e} 8 \mathrm{f}$ of the Protein Data Bank, using the usual structure factor formula 


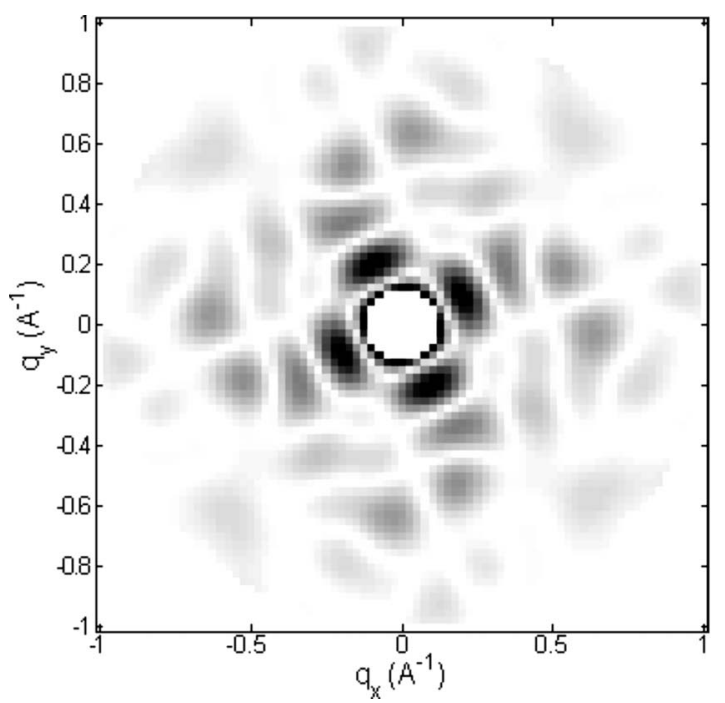

FIG. 1. Amplitudes of a simulated diffraction pattern from $\mathrm{x}$ rays incident down the central pore of a single K-channel protein molecule up to about $6 \AA$ resolution. Since the amplitudes of the central pixels of this diffraction pattern and those of Figs. 4-6 are overwhelmingly dominant, they have been removed for easier visibility of higher-resolution amplitudes. The real-space images of Figs. 2 and 3 were calculated from these (phased) amplitudes (with central pixels included). All figures in this paper are displayed by means of a linear gray scale with darker shading representing higher values.

$$
F\left(q_{x}, q_{y}, \omega_{0}\right)=\sum_{j} f_{j}\left(q_{x}, q_{y}\right) e^{i\left(q_{x} x_{j}+q_{y} y_{j}\right)},
$$

where $f_{j}\left(q_{x}, q_{y}\right)$ is the form factor of an atom $j$ whose coordinates projected onto a plane perpendicular to the incident beam are $\left(x_{j}, y_{j}\right),\left(q_{x}, q_{y}\right)$ are the corresponding twodimensional reciprocal-space coordinates, and $\omega_{0}$ represents the particular molecular orientation assumed for these simulations. The projected electron density (Fig. 2) to the resolution of the diffraction pattern was then computed from the inverse Fourier transform of $\left|F\left(q_{x}, q_{y} ; \omega_{0}\right)\right|$ (calculated up to a maximum value of $q=\sqrt{q_{x}^{2}+q_{y}^{2}}$ of $2.0 \AA^{-1}$ but multiplied by an apodizing Gaussian window function to reduce artifacts) with phases computed by an iterative phasing algorithm. ${ }^{12,16}$

Figure 3 shows the real-space image reconstructed by such an algorithm with information only about the angular pair correlations (4) of the diffraction pattern of Fig. 1.

We next simulated 10000 diffraction patterns each containing 10 such randomly oriented model molecules. The theory of Sec. II shows that, if a sufficient number of DPs are averaged over in Eq. (4), the result of Eq. (7) is obtained for all random positions and orientations of the individual particles. For the parameters of our simulation, interference fringes from interparticle interference are smaller than a detector pixel and could therefore be neglected. Therefore, although the simulations that follow assume a different set of random particle orientations per DP, they make the assumption that it is not necessary to simulate random interparticle vectors, and that each DP may be simulated by

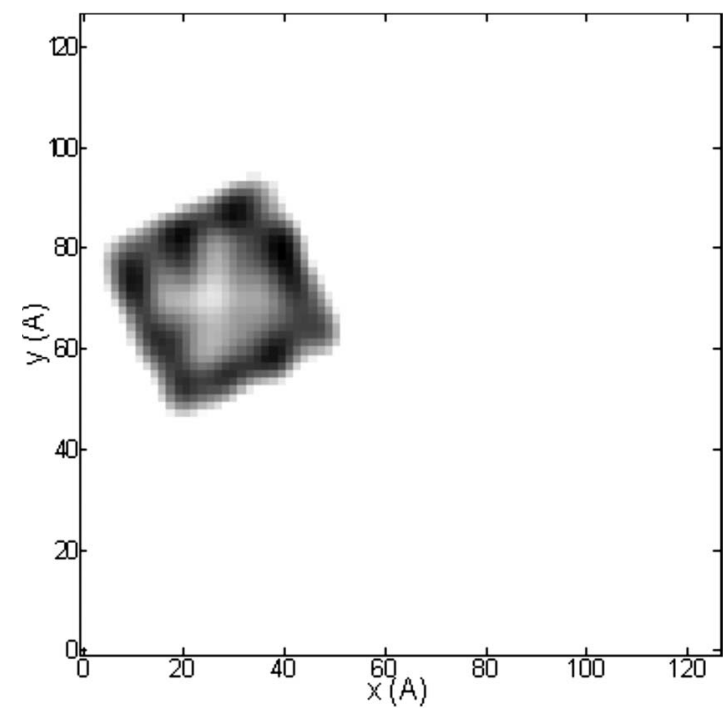

FIG. 2. Electron density of the K-channel protein projected in the direction parallel to its central pore as calculated from a Fourier transform of the scattering amplitudes of Fig. 1, with phases from an iterative phasing algorithm.

$$
I\left(q_{x}, q_{y}\right)=\sum_{k}\left|F_{k}\left(q_{x}, q_{y} ; \omega_{k}\right)\right|^{2}
$$

where, as before, $\omega_{k}$ is a random molecular orientation. A typical pattern from 10 such randomly positioned K-channel proteins, randomly oriented about the $z$ axis [normal to the $(x, y)$ plane] is shown in Fig. 4.

We seek to demonstrate the recovery of the model projected electron density of a single protein from a large number of diffraction patterns of the form of Fig. 4, each from different random orientations of 10 molecules. We do this in two steps: (1) recovery of a single-particle diffraction pattern from these multiparticle diffraction patterns by the methods

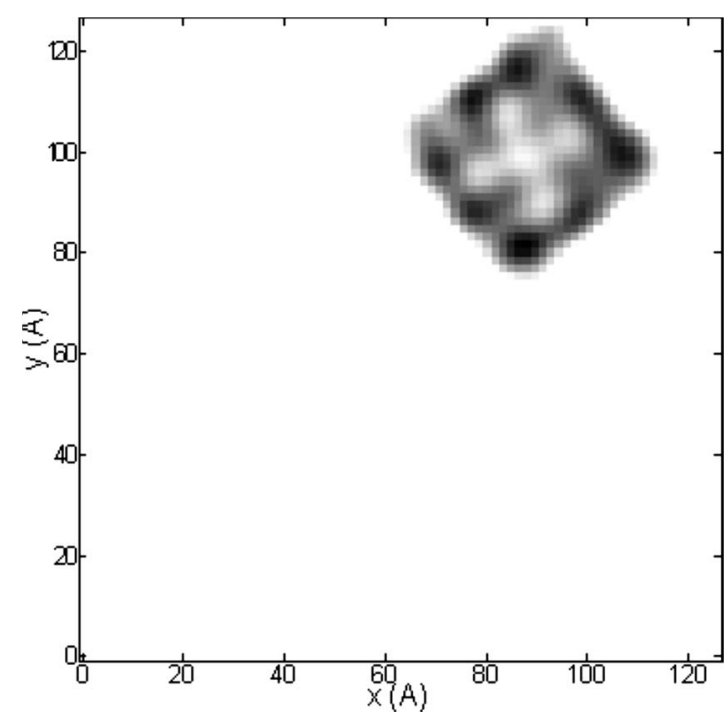

FIG. 3. Same as Fig. 2, except that the projected electron density is calculated from a diffraction pattern recovered from the angular correlations from Fig. 1 and iterative phasing. 


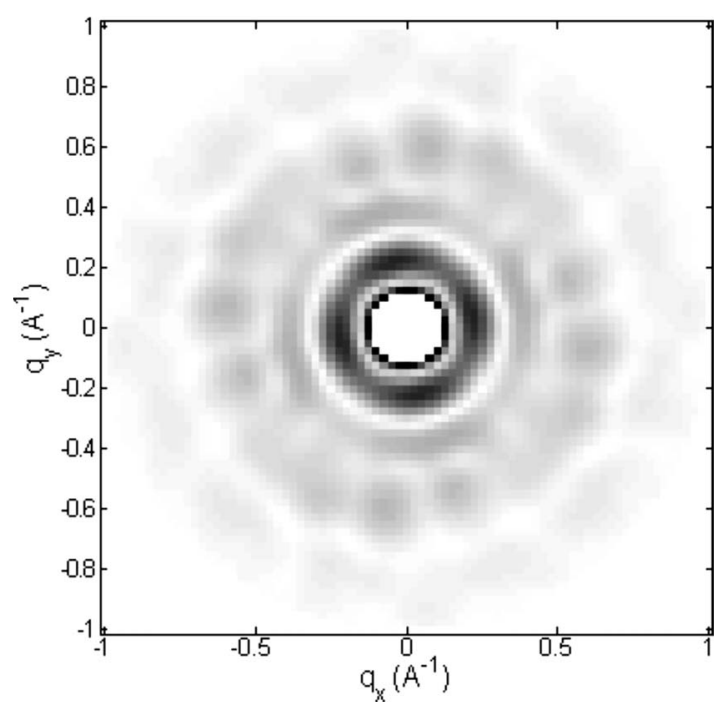

FIG. 4. Typical simulated diffraction pattern from 10 randomly positioned and oriented model K-channel proteins.

described in Secs. II and III above and (2) the recovery of the projected electron density from this by a conventional iterative phasing algorithm.

The recovered single-particle diffraction pattern from the average of the angular correlations of 10000 such diffraction patterns is shown in Fig. 5. Simulated Poisson noise produces an unimportant peak at the origin of the correlation functions $C_{2}$.

\section{FROM RECONSTRUCTED DIFFRACTION PATTERN TO PROJECTED ELECTRON DENSITY}

Finally, we recover (Fig. 6) from the reconstructed diffraction pattern of Fig. 5 the projected electron density of an individual protein molecule after $\sim 100$ iterations of the

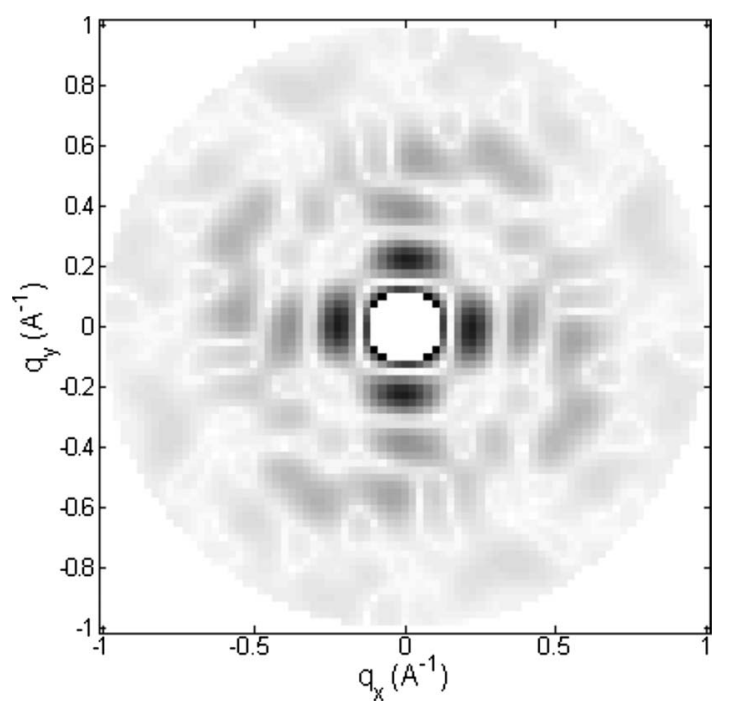

FIG. 5. A single particle diffraction pattern reconstructed from the average of the angular correlations of intensities of 10000 diffraction patterns of the form of Fig. 4.

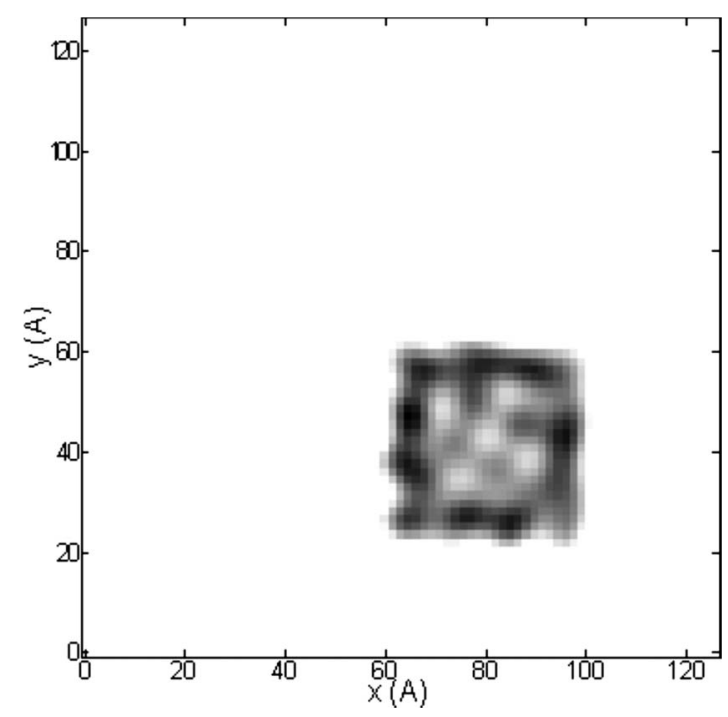

FIG. 6. Projected electron density of a single molecule of the K-channel membrane protein reconstructed from the diffraction pattern of Fig. 5 by an iterative phasing algorithm.

charge-flipping ${ }^{12}$ and phase-shifting algorithms, ${ }^{16}$ which do not impose a fixed region of compact support. ${ }^{17}$

\section{DISCUSSION}

The present paper suggests an alternative route to molecular structure determination than diffraction from either crystals or single molecules. For a given probe size at the sample, by simultaneously scattering off several copies of the molecule, the number of scattered photons per detector pixel is increased substantially compared to single-molecule diffraction experiments. Yet, at the same time, the relaxation of the condition of molecular alignment permits application to molecules, such as many membrane proteins, which do not crystallize.

We demonstrate that averaging the angular correlation functions of many diffraction patterns each from many randomly oriented copies of the molecule allows the reconstruction of a diffraction pattern of a single molecule of sufficient quality to allow an iterative phasing algorithm to reconstruct an accurate real-space image of the molecule.

The Fourier transform of the autocorrelation functions of each resolution ring yields the magnitudes $\left|I_{M}(q)\right|$ of the circular harmonic expansion coefficients of the intensities. The earlier method of finding the phases of these coefficients relied on the evaluations also of so-called angular triple correlation functions. ${ }^{4,11}$ It was found ${ }^{11}$ that the construction of converged triple correlation functions required the averaging of data from many more measured diffraction patterns than required to construct converged angular pair correlation functions. The algorithm presented here needs the experimental data of only the angular pair correlations, as the phases of these coefficients are found directly from their magnitudes as in algorithms for the usual phase problem in the for the recovery of an object's electron density from the magnitudes of its diffracted intensities. The liberation from the need to measure a much larger number of diffraction 
patterns to obtain converged triple correlations makes for a much less costly experiment due to need for less beamtime. The computational requirements for finding the phases of $I_{M}(q)$ by an iterative phasing algorithm are also much reduced compared to the use of, e.g., a simulated annealing algorithm to optimize the agreement between experimental and theoretical triple correlations, as previously.

The results are also relevant also to the kind of "diffract and destroy" approach to biomolecular structure determination $^{18-20}$ with an $\mathrm{x}$-ray free electron laser, where measurements of diffraction from single molecules have been proposed. In this case, an average of the angular correlation functions over many such very weak diffraction patterns allows a building up of a more statistically reliable signal from which it has been proposed that the diffraction volume of an individual molecule may be built up. ${ }^{9}$ Since the number of values of the angular correlation functions do not grow as the data of more diffraction patterns are allowed to contribute the average, this would also be expected to provide an efficient method of data reduction in the millions of very weak diffraction patterns that are typically measured in such experiments. In addition, the SAXS background, which arises from uncorrelated scattering by different particles, and which needs to be subtracted out in the present treatment, is entirely absent, as is scattering from membrane or solution atoms.

The method may be applicable also to x-ray fluorescence patterns from a specific atomic species buried deeply within large molecules ${ }^{21}$ (e.g., the Fe atom in hemoglobin) in solution. Such patterns would be most sensitive to intramolecular scattering, less to that from its surroundings. This may allow the reconstruction of the $3 \mathrm{D}$ diffraction volume of an individual molecule from that of many randomly oriented ones, and hence of the molecular structure, without too much interference from solvent (or membrane) scattering.

\section{CONCLUSIONS}

Advances in technology, such as fast column read-out area detectors, brighter sources, shorter pulses, and zoneplate focusing, have greatly improved the experimental conditions for such experiments. This has been demonstrated recently by Wochner et al., ${ }^{22}$ who suggested the possible use of angular correlation measurements to reveal hidden symmetries of short-range order which are not apparent in the typical ring diffraction patterns of disordered matter from which what is usually analyzed is just the radial intensity distribution $I(q)$, otherwise known as the SAXS data. The present paper shows how information about not just the local symmetries but even the detailed structure of the microscopic structural units may be found from the angular correlations.

\section{ACKNOWLEDGMENTS}

We thank Marius Schmidt for help with generating an atomic model of the K-channel membrane protein and Vali Raicu for helpful discussions. D.K.S. and J.C.H.S. jointly acknowledge support from DOE under Grant No. DESC0002141, D.K.S. from DOE Grant No. DE-FG02-06ER46277 and the Research Growth Initiative of the University of Wisconsin-Milwaukee.

\footnotetext{
*Deceased.

${ }^{1}$ M. R. Howells et al., J. Electron Spectrosc. Relat. Phenom. 170, 4 (2009).

${ }^{2}$ S. Marchesini, Rev. Sci. Instrum. 78, 049901 (2007).

${ }^{3}$ Z. Kam, Macromolecules 10, 927 (1977).

${ }^{4}$ Z. Kam, J. Theor. Biol. 82, 15 (1980).

${ }^{5}$ H. B. Stuhrmann, Acta Crystallogr., Sect. A: Cryst. Phys., Diffr., Theor. Gen. Crystallogr. 26, 297 (1970).

${ }^{6}$ D. I. Svergun and H. B. Stuhrmann, Acta Crystallogr., Sect. A: Found. Crystallogr. 47, 736 (1991).

${ }^{7}$ V. L. Shneerson and D. K. Saldin, Acta Crystallogr., Sect. A: Found. Crystallogr. 65, 128 (2009).

${ }^{8}$ D. I. Svergun and M. H. Koch, Curr. Opin. Struct. Biol. 12, 654 (2002).

${ }^{9}$ D. K. Saldin, V. L. Shneerson, R. Fung, and A. Ourmazd, J. Phys.: Condens. Matter 21, 134014 (2009).

${ }^{10}$ J. Karle and H. Hauptman, Acta Crystallogr. 3, 181 (1950).

${ }^{11}$ D. K. Saldin et al., New J. Phys. 12, 035014 (2010).

${ }^{12}$ G. Oszlányi and A. Süto, Acta Crystallogr., Sect. A: Found. Crystallogr. 60, 134 (2004).
}

${ }^{13}$ A. V. Oppenheim, R. W. Schafer, and J. R. Buck, Discrete-Time Signal Processing (Prentice-Hall, Upper Saddle River, 1999).

${ }^{14}$ H. C. Poon and D. K. Saldin (unpublished).

${ }^{15}$ D. A. Doyle, J. M. Cabral, R. A. Pfeutzner, A. Kuo, J. M. Gulbis, S. L. Cohen, B. T. Chait, and R. MacKinnon, Science 280, 69 (1998).

${ }^{16}$ G. Oszlányi and A. Süto, Acta Crystallogr., Sect. A: Found. Crystallogr. 61, 147 (2005).

${ }^{17}$ J. R. Fienup, Appl. Opt. 21, 2758 (1982).

${ }^{18}$ R. Neutze, R. Wouts, D. van der Spoel, E. Weckert, and J. Hajdu, Nature (London) 406, 752 (2000).

${ }^{19}$ V. L. Shneerson, A. Ourmazd, and D. K. Saldin, Acta Crystallogr., Sect. A: Found. Crystallogr. 64, 303 (2008).

${ }^{20}$ R. Fung, V. L. Shneerson, D. K. Saldin, and A. Ourmazd, Nat. Phys. 5, 64 (2009).

${ }^{21}$ G. Faigel, G. Bortel, C. S. Fadley, A. S. Simionovici, and M. Tegze, X-Ray Spectrom. 36, 3 (2007).

${ }^{22}$ P. Wochner, C. Gutt, T. Autenreith, T. Demmer, V. Bugaev, A. D. Ortiz, A. Duri, F. Zontone, G. Greubel, and H. Dosch, Proc. Natl. Acad. Sci. U.S.A. 106, 11511 (2009). 